Original article

\title{
Socio-economic factors associated with increased neonatal mortality: A mixed-method study of Bangladesh and 20 other developing countries based on demographic and health survey data
}

\author{
Md. Akhtarul Islam ${ }^{\text {a, *, Biva Biswas }}{ }^{\text {a }}$ \\ ${ }^{\text {a }}$ Statistics Discipline, Science, Engineering and Technology (SET) School, Khulna University, Khulna, 9208, Bangladesh
}

\section{A R T I C L E I N F O}

\section{Keywords:}

Neonatal mortality

Antenatal care

Developing countries

SDGs

Meta-analysis

\begin{abstract}
A B S T R A C T
Background: Neonatal mortality is a significant pointer of a country's level of socio-economic development and quality of life. The neonatal mortality rate reduction is an important goal of Sustainable Development Goals (SDGs) as it is decreasing slowly but surely compare to under-five mortality in developing countries. This study desired to determine the prevalence of neonatal mortality and its associated risk factors in developing countries. Methods: This study used the latest DHS data of 21 different developing countries, including Bangladesh. Logistic regression model and Meta -Analysis techniques were used in this study.

Results: A logistic regression model reveals that, for Bangladesh mother's higher-level education (OR: 0.591; CI: 0.401-0.872)and father's higher-level education (OR: 0.352; CI: 0.135-0.915), better wealth status (OR: 0.467; CI: 0.275-0.795), being 2nd born and above order neonate (OR: 0.329; CI: 0.216-0.501), above 19 years for delivery (OR: 0.51; CI: 0.341-0.763), being female neonate (OR: 0.352; CI: 0.253-0.49), ANC visit, (OR: 0.206; CI: 0.144-0.296), media access (OR: 0.590; CI: 0.391-0.891) were statistically significant predictors of lowering neonatal death. While being twin birth (OR: 8.815; CI: 4.057-19.153) is revealed as a risk factor of neonatal mortality. These results were justified by meta-analyses for the developing countries as well.

Conclusion: In this study, mother's and father's lower education level, poor wealth status, being 1st born neonate, early maternal age, being male neonate, being twin birth, having no ANC visit, no media access was found to be statistically significant influencing factors of neonatal death in developing countries.
\end{abstract}

Key points

The number of fetus and the size of the child were positively associated with neonatal mortality but, utilization of ANC, maternal, and paternal education were negatively associated with neonatal mortality in 21 developing countries. This study recommends increasing ANC utilization, ensuring maternal and paternal education to raise awareness regarding neonatal mortality to achieve the targeted goal of reducing neonatal mortality by the Sustainable Development Goal by 2030 .

\section{What is new?}

Using the DHS data of 21 developing countries, this study has revealed the socio-economic influencing factors and their consistency across the developing countries. This study also showed a mixed-method analysis and compared the logistic regression model results with meta- analysis results to get more precise results.

\section{Introduction}

The most vulnerable time of human life for diseases is the neonatal period, which is defined as the duration of the first four weeks or 28 days from birth. ${ }^{1,2}$ In this period, the risk of dying of a child is almost 15 times greater than at any other time during the first year of life. ${ }^{3}$ Although the total annual deaths of children younger than five years are reducing, the relative magnitude of neonatal death is increasing, and the portion of neonatal death of total yearly deaths of children younger than five years is about $45 \%{ }^{4}$ Approximately $98 \%$ of total neonatal death occurs in low-income and middle-income countries, especially in sub -Saharan African and South Asian. ${ }^{4-6}$ Among ten countries with the highest neonatal mortality rate, eight sub-Saharan and 2 are south Asian. In sub-Saharan Africa, 1.2 million newborns died every year alone, which

\footnotetext{
* Corresponding author. Statistics Discipline, Science, Engineering and Technology (SET) School, Khulna University, Khulna, 9208, Bangladesh.

E-mail address: akhtarulstat@ku.ac.bd (Md.A. Islam).
} 

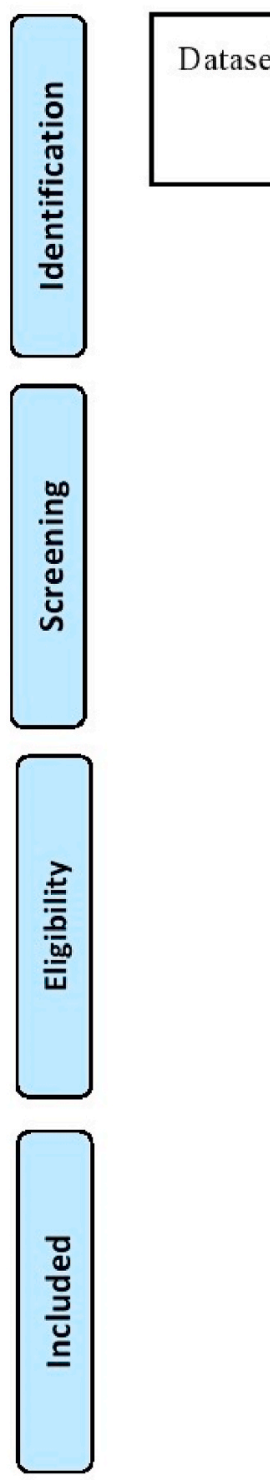

Additional datasets/records identified through other sources database searching $(\mathrm{n}=91)$

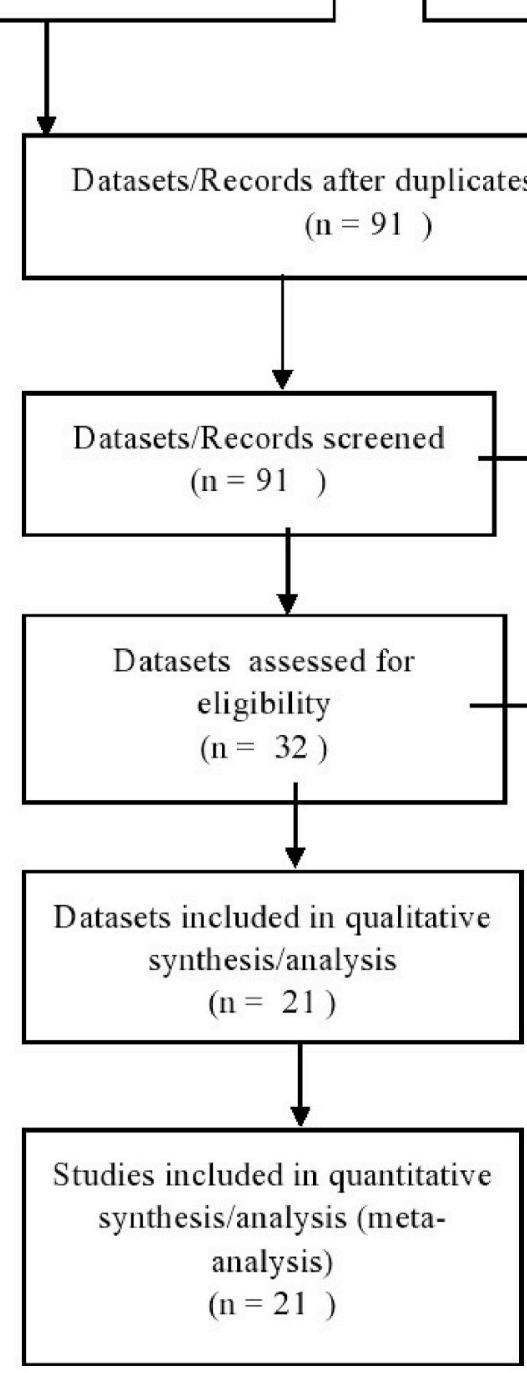

$$
(\mathrm{n}=0)
$$

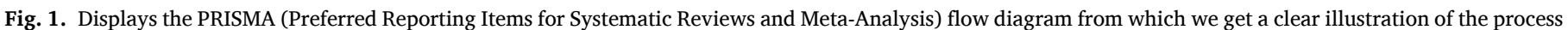
of identifying and including DHS datasets for the random effect meta-analysis.

is equivalent to 13000 deaths per day or nine deaths every minute almost . This neonatal death is influenced by multiple factors and represents a complex interaction among these variables. ${ }^{8}$ Biological factors, socio-economic status, demographics factors, health care system, cultural practices, and technologies are essential determinants of neonatal death. ${ }^{9,10}$ Even a countries socio-economic condition efficiency and effectiveness of healthcare systems as well as achieving optimal education can be standardized by the neonatal morbidity and mortality rates. ${ }^{11}$ Neonatal death is mostly preventable, and up to two-third of this death can be reduced with the provision of essential newborn care at birth and during the neonatal period. ${ }^{12}$ In developed countries, the neonatal mortality rate has been reduced using develop health facility appropriate techniques and proper steps. ${ }^{13,14}$ However, the neonatal mortality rate is still unacceptably high at 37 per 1000 life birth exceeding the global estimate in low-income and middle-income countries. $^{15}$
To improve the overall condition, the world introduced the sustainable development goals (SDGs) after millennium development goals (MDGs) in 2015, keeping the maternal, neonatal, child, and adolescent health issues in central. ${ }^{16}$ In SDGs, the first three targets of health goals are the continuations of MDGs. The important one is to reduce neonatal mortality to below 12 deaths per 1000 live births and under-five mortality to below 25 deaths per 1000 live births. ${ }^{1,17}$ To achieve the SDGs first, it is essential to explore the factors that contribute to neonatal death. Identifying the cause of neonatal death and triggering factors of neonatal mortality will help to select appropriate strategies to reduce the neonatal mortality rate. ${ }^{18}$ In our study, considering the vital facts related to neonatal mortality, we tried to find out the socio-economic and demographic influencing factors of neonatal mortality and their consistency across the 21 developing countries, including Bangladesh. We have introduced a mixed method that was designated to combine data from Bangladesh and 20 other countries, which we think would be more 
effective to find out more precious summary results of the influencing factors of neonatal death than using a simple cross-sectional survey and meta-analysis.

\section{Materials and methods}

\subsection{Design}

We applied a mixed-method design to conduct the study comprising both binary logistic regression for the cross-sectional survey of Bangladesh and meta-analysis for 21 developing countries. Afterward, we made a comparison between the results of Logistic regression of Bangladesh and the findings of a meta-analysis of 21 developing countries. We could broadly explore the influential determinants of neonatal mortality by employing this approach. All the data were taken from the Demographic and Health Survey (DHS).

\subsection{Data source and data extraction}

Initially, for this cross-sectional study, we extracted relevant information for analysis from a nationwide representative secondary dataset, Bangladesh Demography and Health Survey 2014, for binary logistic regression. ${ }^{19}$ Besides, we conducted a meta-analysis utilizing the recently accessible datasets (accessed in July 2019) from MEASURE DHS (Monitoring and evaluating to Assess and Use Result, Demographic and Health Survey) (www.measuredhs.com). We adopted the recent available DHS data for the other 20 developing countries. ${ }^{20}$ Which are Afghanistan (2015), Angola (2015-16), Benin (2017-18), Chad (2014-15), Cambodia (2014), Ethiopia (2016), Ghana (2014), Guinea (2018), India (2015-16), Indonesia (2017), Kenya (2014), Lesotho (2014), Myanmar (2015-16), Nepal (2016), Nigeria (2018), Pakistan (2017-18), Sierra Leone (2013), Timor-Leste (2016), Zambia (2013-14), Zimbabwe (2015). DHS program provides high-quality, accessible data for analysis in the form of a questionnaire, which was used as one of the elementary goals of the DHS program. The DHS database contains information from 91 countries (http://dhsprogram.co $\mathrm{m}$ /data/available-datasets.com), but 70 countries were excluded due to excessive missing values, developing countries' checklist, and unavailability of information regarding independent variable in any of the selected countries. Finally, we have chosen Bangladesh along with 20 developing countries that are homogeneous in nature as similar probability sampling for data collection was applied to them (Fig. 1).

\section{Variables}

In this study, we considered neonatal mortality as the dependent variable. The outcome variable, "Neonatal mortality," was recorded as a binary variable. If the live-born infant dies with the first four weeks of its life, we recorded it as "Yes," otherwise "No." We included different relevant socio-economic and demographic factors as independent variables to execute the research and to discover the triggering factors of the neonatal mortality based on the previous literature. ${ }^{5,12,18,21,22}$ We used the standard covariates whose data were available for all selected countries. Commencing with the type of place of residence, sex of the child, and mother working status remained the same as the existing category of DHS datasets. The remaining covariates were subcategorized. We merged no education and primary to up to primary, secondary, and higher in terms of parent's education level for BDHS data. Again, for meta-analysis, we combined secondary and higher to above primary while another category was up to the primary. In the case of binary logistic regression, the wealth index has remained the same as the original data. Further, we changed the label of the variable to Living below the poverty line for the meta-analysis with two categories. We combined poorer and poorest and labeled them as 'yes', which means if the individuals are poor, they certainly live below the poverty line; on the other hand, we combined middle, richer, and richest with the label
Table 1

Frequency distribution of different variables with levels for BDHS data.

\begin{tabular}{|c|c|c|c|}
\hline Variables & Categories & Frequencies & Percentages \\
\hline \multicolumn{4}{|l|}{ Dependent variables } \\
\hline \multirow[t]{2}{*}{ Neonatal mortality } & No & 4586 & 973 (in 1000) \\
\hline & Yes & 127 & 27 (in 1000) \\
\hline \multicolumn{4}{|l|}{ Explanatory variables } \\
\hline \multirow[t]{2}{*}{ Maternal age at delivery } & Up to 19 years & 1166 & 24.7 \\
\hline & Above 19 years & 3547 & 75.3 \\
\hline \multirow[t]{2}{*}{ Type of place of residence } & Rural & 3210 & 68.1 \\
\hline & Urban & 1503 & 31.9 \\
\hline \multirow[t]{3}{*}{ Mother's education level } & Up to primary & 1954 & 41.5 \\
\hline & Secondary & 2219 & 47.1 \\
\hline & Higher & 540 & 11.4 \\
\hline \multirow[t]{2}{*}{ Mass media } & No & 1811 & 38.4 \\
\hline & Yes & 2902 & 61.6 \\
\hline \multirow[t]{5}{*}{ Wealth Index } & Poorest & 1009 & 21.4 \\
\hline & Poorer & 897 & 19.0 \\
\hline & Middle & 899 & 19.1 \\
\hline & Richer & 987 & 20.8 \\
\hline & Richest & 930 & 19.7 \\
\hline \multirow[t]{3}{*}{ Father's education level } & Up to primary & 2522 & 53.5 \\
\hline & Secondary & 1472 & 31.2 \\
\hline & Higher & 719 & 15.3 \\
\hline \multirow{2}{*}{ Mother's working status } & No & 3682 & 78.1 \\
\hline & Yes & 1031 & 21.9 \\
\hline \multirow[t]{2}{*}{ Birth order number } & 1st born & 1938 & 41.1 \\
\hline & 2nd and above & 2775 & 58.9 \\
\hline \multirow[t]{2}{*}{ Number of fetus } & Single & 4651 & 98.7 \\
\hline & One of multiple & 62 & 1.3 \\
\hline \multirow[t]{2}{*}{ Sex of child } & Male & 2431 & 51.6 \\
\hline & Female & 2282 & 48.4 \\
\hline \multirow[t]{2}{*}{ Place of delivery } & Home & 2857 & 60.6 \\
\hline & Hospital & 1856 & 39.4 \\
\hline \multirow[t]{2}{*}{ Antenatal care utilization } & No & 1203 & 25.5 \\
\hline & Yes & 3510 & 74.5 \\
\hline \multirow[t]{3}{*}{ Size of child } & Average & 3173 & 67.3 \\
\hline & Larger than average & 614 & 13.0 \\
\hline & Smaller than average & 926 & 19.6 \\
\hline
\end{tabular}

'no,' which represents the individuals who live above the poverty line. The variable birth order number was categorized as firstborn and 2nd and above born for both binary logistic regression and meta-analysis. Maternal age at delivery was converted into a nominal scale from the continuous form with the category less than or equal to 19 years $(\leq 19)$ and above 19 years. $^{9}$ Categories for the variable number of the fetus were single and multiple for both methods. For binary logistic regression, we subcategorized taking antenatal care (ANC) as no visits, 1-4 visits, more than four visits, whereas, in the meta-analysis, individuals who had at least one ANC visit were considered in the category yes, otherwise no. For the variable mass media, we categorized it as 'yes', which represents access to mass media, and 'no' represents no media access. Variable place of delivery was categorized as Home and Hospital for both logistic regression and meta-analysis. Finally, for the size of the child, we subcategorized the variable as average, larger than average (combined by very large and larger than average), and smaller than average (combined by very small and smaller than average) for the logistic regression analysis. We further recoded this variable for meta-analysis with two independent levels, such as average and larger or smaller than average.

\subsection{Statistical analysis}

We used statistical software SPSS V.23 (SPSS Inc. Chicago, USA) and R V.3.6.2 (Bell Laboratories, New Jersey, USA) to carry out the analysis. Binary logistic regression was practiced determining the key factors that have an impact on neonatal mortality in Bangladesh using BDHS data. ${ }^{23}$ Besides, we applied meta-analysis on the DHS data from 21 developing countries. Heterogeneity was assessed by enumerating values from $\mathrm{I}^{2}$ and $\mathrm{p}$ values among datasets. ${ }^{24}$ We performed a random-effects model in the meta-analytical approach as significant heterogeneity was found by 
Table 2

Different categories of variables and frequency distribution for 21 developing countries DHS data.

\begin{tabular}{|c|c|c|c|c|c|c|c|c|c|c|c|c|c|c|c|}
\hline \multirow{3}{*}{$\begin{array}{r}\text { Country } \\
\text { Name }\end{array}$} & \multicolumn{15}{|l|}{ Variable } \\
\hline & \multicolumn{2}{|c|}{$\begin{array}{l}\text { Neonatal } \\
\text { mortality (in } \\
1000 \text { ) }\end{array}$} & \multicolumn{3}{|c|}{$\begin{array}{l}\text { Maternal Age at } \\
\text { Delivery n (\%) }\end{array}$} & \multicolumn{2}{|c|}{ Residence n (\%) } & \multicolumn{2}{|c|}{$\begin{array}{l}\text { Mother's Education } \mathrm{n} \\
\text { (\%) }\end{array}$} & \multicolumn{2}{|c|}{$\begin{array}{l}\text { Media Access n } \\
(\%)\end{array}$} & \multicolumn{2}{|c|}{$\begin{array}{l}\text { Living below } \\
\text { Poverty Line n (\%) }\end{array}$} & \multicolumn{2}{|c|}{$\begin{array}{l}\text { Father's Education } n \\
(\%)\end{array}$} \\
\hline & No & Yes & $\begin{array}{l}\leq 19 \\
\text { years }\end{array}$ & $\mathrm{y}$ & & Rural & Urban & $\begin{array}{l}\text { Up to } \\
\text { primary }\end{array}$ & $\begin{array}{l}\text { Above } \\
\text { primary }\end{array}$ & No & Yes & No & Yes & $\begin{array}{l}\text { Up to } \\
\text { primary }\end{array}$ & $\begin{array}{l}\text { Above } \\
\text { primary }\end{array}$ \\
\hline $\begin{array}{l}\text { Sierra Leone } \\
2013\end{array}$ & $\begin{array}{l}9836 \\
(968)\end{array}$ & $\begin{array}{l}328 \\
(32)\end{array}$ & $\begin{array}{l}1143 \\
(11.2)\end{array}$ & $\begin{array}{l}90 \\
(8\end{array}$ & & $\begin{array}{l}7377 \\
(72.6)\end{array}$ & $\begin{array}{l}2787 \\
(27.4)\end{array}$ & $\begin{array}{l}8828 \\
(86.9)\end{array}$ & $\begin{array}{l}1336 \\
(13.1)\end{array}$ & $\begin{array}{l}4287 \\
(42.2)\end{array}$ & $\begin{array}{l}5877 \\
(57.8)\end{array}$ & $\begin{array}{l}4613 \\
(45.4)\end{array}$ & $\begin{array}{l}5551 \\
(54.6)\end{array}$ & $\begin{array}{l}7616 \\
(74.9)\end{array}$ & $\begin{array}{l}2548 \\
(25.1)\end{array}$ \\
\hline $\begin{array}{l}\text { Zambia } \\
2013-14\end{array}$ & $\begin{array}{l}11318 \\
(977)\end{array}$ & $\begin{array}{l}263 \\
(23)\end{array}$ & $\begin{array}{l}1231 \\
(10.6)\end{array}$ & & $\begin{array}{l}50 \\
4)\end{array}$ & $\begin{array}{l}7389 \\
(63.8)\end{array}$ & $\begin{array}{l}4192 \\
(36.2)\end{array}$ & $\begin{array}{l}8026 \\
(69.3)\end{array}$ & $\begin{array}{l}3555 \\
(30.7)\end{array}$ & $\begin{array}{l}3836 \\
(33.1)\end{array}$ & $\begin{array}{l}7745 \\
(66.9)\end{array}$ & $\begin{array}{l}5607 \\
(48.4)\end{array}$ & $\begin{array}{l}5974 \\
(51.6)\end{array}$ & $\begin{array}{l}5719 \\
(49.4)\end{array}$ & $\begin{array}{l}5862 \\
(59.6)\end{array}$ \\
\hline $\begin{array}{l}\text { Bangladesh } \\
2014\end{array}$ & $\begin{array}{l}4586 \\
(973)\end{array}$ & $\begin{array}{l}127 \\
(27)\end{array}$ & $\begin{array}{l}1166 \\
(24.7)\end{array}$ & $\begin{array}{l}35 \\
(7\end{array}$ & & $\begin{array}{l}3210 \\
(68.1)\end{array}$ & $\begin{array}{l}1503 \\
(31.9)\end{array}$ & $\begin{array}{l}1954 \\
(41.5)\end{array}$ & $\begin{array}{l}2759 \\
(58.5)\end{array}$ & $\begin{array}{l}1811 \\
(38.4)\end{array}$ & $\begin{array}{l}2902 \\
(61.6)\end{array}$ & $\begin{array}{l}1906 \\
(40.4)\end{array}$ & $\begin{array}{l}2807 \\
(59.6)\end{array}$ & $\begin{array}{l}2522 \\
(53.5)\end{array}$ & $\begin{array}{l}2191 \\
(46.5)\end{array}$ \\
\hline $\begin{array}{l}\text { Cambodia } \\
2014\end{array}$ & $\begin{array}{l}6981 \\
(987)\end{array}$ & $\begin{array}{l}94 \\
(13)\end{array}$ & $\begin{array}{l}491 \\
(6.9)\end{array}$ & $\begin{array}{l}65 \\
(9\end{array}$ & & $\begin{array}{l}5147 \\
(72.7)\end{array}$ & $\begin{array}{l}1928 \\
(27.3)\end{array}$ & $\begin{array}{l}4503 \\
(63.6)\end{array}$ & $\begin{array}{l}2572 \\
(36.4)\end{array}$ & $\begin{array}{l}1459 \\
(20.6)\end{array}$ & $\begin{array}{l}5616 \\
(79.4)\end{array}$ & $\begin{array}{l}3005 \\
(42.5)\end{array}$ & $\begin{array}{l}4070 \\
(57.5)\end{array}$ & $\begin{array}{l}3731 \\
(52.7)\end{array}$ & $\begin{array}{l}3344 \\
(47.3)\end{array}$ \\
\hline Ghana 2014 & $\begin{array}{l}5240 \\
(973)\end{array}$ & $\begin{array}{l}144 \\
(27)\end{array}$ & $\begin{array}{l}260 \\
(4.8)\end{array}$ & $\begin{array}{l}51 \\
(9\end{array}$ & & $\begin{array}{l}3269 \\
(60.7)\end{array}$ & $\begin{array}{l}2115 \\
(39.3)\end{array}$ & $\begin{array}{l}3078 \\
(57.2)\end{array}$ & $\begin{array}{l}2306 \\
(42.8)\end{array}$ & $\begin{array}{l}726 \\
(13.5)\end{array}$ & $\begin{array}{l}4658 \\
(86.5)\end{array}$ & $\begin{array}{l}2962 \\
(55.0)\end{array}$ & $\begin{array}{l}2422 \\
(45.0)\end{array}$ & $\begin{array}{l}2310 \\
(42.9)\end{array}$ & $\begin{array}{l}3074 \\
(57.1)\end{array}$ \\
\hline Kenya 2014 & $\begin{array}{l}9007 \\
(980)\end{array}$ & $\begin{array}{l}184 \\
(20)\end{array}$ & $\begin{array}{l}780 \\
(8.5)\end{array}$ & $\begin{array}{l}84 \\
(9\end{array}$ & & $\begin{array}{l}6168 \\
(67.1)\end{array}$ & $\begin{array}{l}3023 \\
(32.9)\end{array}$ & $\begin{array}{l}6973 \\
(75.9)\end{array}$ & $\begin{array}{l}2218 \\
(24.1)\end{array}$ & $\begin{array}{l}2380 \\
(25.9)\end{array}$ & $\begin{array}{l}6811 \\
(74.1)\end{array}$ & $\begin{array}{l}5056 \\
(55.0)\end{array}$ & $\begin{array}{l}4135 \\
(45.0)\end{array}$ & $\begin{array}{l}6137 \\
(66.8)\end{array}$ & $\begin{array}{l}3054 \\
(33.2)\end{array}$ \\
\hline Lesotho 2014 & $\begin{array}{l}2635 \\
(966)\end{array}$ & $\begin{array}{l}94 \\
(34)\end{array}$ & $\begin{array}{l}314 \\
(11.5)\end{array}$ & $\begin{array}{l}24 \\
(8\end{array}$ & & $\begin{array}{l}2071 \\
(75.9)\end{array}$ & $\begin{array}{l}658 \\
(24.1)\end{array}$ & $\begin{array}{l}1408 \\
(51.6)\end{array}$ & $\begin{array}{l}1321 \\
(48.4)\end{array}$ & $\begin{array}{l}762 \\
(27.9)\end{array}$ & $\begin{array}{l}1967 \\
(72.1)\end{array}$ & $\begin{array}{l}1303 \\
(47.7)\end{array}$ & $\begin{array}{l}1426 \\
(52.3)\end{array}$ & $\begin{array}{l}1784 \\
(65.4)\end{array}$ & $\begin{array}{l}945 \\
(34.6)\end{array}$ \\
\hline $\begin{array}{l}\text { Chad } \\
\text { 2014-15 }\end{array}$ & $\begin{array}{l}16290 \\
(973)\end{array}$ & $\begin{array}{l}454 \\
(27)\end{array}$ & $\begin{array}{l}2575 \\
(15.4)\end{array}$ & & $\begin{array}{l}69 \\
.6)\end{array}$ & $\begin{array}{l}13274 \\
(79.3)\end{array}$ & $\begin{array}{l}3470 \\
(20.7)\end{array}$ & $\begin{array}{l}15393 \\
(91.9)\end{array}$ & $\begin{array}{l}1351 \\
(8.1)\end{array}$ & $\begin{array}{l}12598 \\
(75.2)\end{array}$ & $\begin{array}{l}4146 \\
(24.8)\end{array}$ & $\begin{array}{l}6560 \\
(39.2)\end{array}$ & $\begin{array}{l}10184 \\
(60.8)\end{array}$ & $\begin{array}{l}13746 \\
(82.1)\end{array}$ & $\begin{array}{l}2998 \\
(17.9)\end{array}$ \\
\hline $\begin{array}{l}\text { Afghanistan } \\
2015\end{array}$ & $\begin{array}{l}30087 \\
(977)\end{array}$ & $\begin{array}{l}705 \\
(23)\end{array}$ & $\begin{array}{l}2813 \\
(9.1)\end{array}$ & & $\begin{array}{l}79 \\
9)\end{array}$ & $\begin{array}{l}23384 \\
(75.9)\end{array}$ & $\begin{array}{l}7408 \\
(24.1)\end{array}$ & $\begin{array}{l}28524 \\
(92.6)\end{array}$ & $\begin{array}{l}2268 \\
(7.4)\end{array}$ & $\begin{array}{l}12263 \\
(39.8)\end{array}$ & $\begin{array}{l}18529 \\
(60.2)\end{array}$ & $\begin{array}{l}12637 \\
(41.0)\end{array}$ & $\begin{array}{l}18155 \\
(59.0)\end{array}$ & $\begin{array}{l}21862 \\
(71.0)\end{array}$ & $\begin{array}{l}8930 \\
(29.0)\end{array}$ \\
\hline $\begin{array}{l}\text { Zimbabwe } \\
2015\end{array}$ & $\begin{array}{l}5002 \\
(976)\end{array}$ & $\begin{array}{l}125 \\
(24)\end{array}$ & $\begin{array}{l}509 \\
(9.9)\end{array}$ & $\begin{array}{l}46 \\
(9\end{array}$ & & $\begin{array}{l}3218 \\
(62.8)\end{array}$ & $\begin{array}{l}1909 \\
(37.2)\end{array}$ & $\begin{array}{l}1554 \\
(30.3)\end{array}$ & $\begin{array}{l}3573 \\
(69.7)\end{array}$ & $\begin{array}{l}1268 \\
(24.7)\end{array}$ & $\begin{array}{l}3859 \\
(75.3)\end{array}$ & $\begin{array}{l}1982 \\
(38.7)\end{array}$ & $\begin{array}{l}3145 \\
(61.3)\end{array}$ & $\begin{array}{l}1137 \\
(22.2)\end{array}$ & $\begin{array}{l}3990 \\
(77.8)\end{array}$ \\
\hline $\begin{array}{l}\text { Angola } \\
2015-16\end{array}$ & $\begin{array}{l}9028 \\
(980)\end{array}$ & $\begin{array}{l}183 \\
(20)\end{array}$ & $\begin{array}{l}1214 \\
(13.2)\end{array}$ & $\begin{array}{l}79 \\
(8\end{array}$ & & $\begin{array}{l}4081 \\
(44.3)\end{array}$ & $\begin{array}{l}5130 \\
(55.7)\end{array}$ & $\begin{array}{l}6666 \\
(72.4)\end{array}$ & $\begin{array}{l}2545 \\
(27.6)\end{array}$ & $\begin{array}{l}3285 \\
(35.7)\end{array}$ & $\begin{array}{l}5926 \\
(64.3)\end{array}$ & $\begin{array}{l}4617 \\
(50.1)\end{array}$ & $\begin{array}{l}4594 \\
(49.9)\end{array}$ & $\begin{array}{l}4532 \\
(49.2)\end{array}$ & $\begin{array}{l}4679 \\
(50.8)\end{array}$ \\
\hline $\begin{array}{l}\text { India } \\
\qquad 2015-16\end{array}$ & $\begin{array}{l}42623 \\
(972)\end{array}$ & $\begin{array}{l}1234 \\
(28)\end{array}$ & $\begin{array}{l}2832 \\
(6.5)\end{array}$ & & $\begin{array}{l}25 \\
.5)\end{array}$ & $\begin{array}{l}32883 \\
(75.0)\end{array}$ & $\begin{array}{l}10974 \\
(25.0)\end{array}$ & $\begin{array}{l}19010 \\
(43.3)\end{array}$ & $\begin{array}{l}24847 \\
(56.7)\end{array}$ & $\begin{array}{l}11483 \\
(26.2)\end{array}$ & $\begin{array}{l}32374 \\
(73.8)\end{array}$ & $\begin{array}{l}20798 \\
(47.4)\end{array}$ & $\begin{array}{l}23059 \\
(52.6)\end{array}$ & $\begin{array}{l}14102 \\
(32.2)\end{array}$ & $\begin{array}{l}29755 \\
(67.8)\end{array}$ \\
\hline $\begin{array}{l}\text { Myanmar } \\
2015-16\end{array}$ & $\begin{array}{l}4420 \\
(974)\end{array}$ & $\begin{array}{l}120 \\
(26)\end{array}$ & $\begin{array}{l}218 \\
(4.8)\end{array}$ & $\begin{array}{l}43 \\
(9\end{array}$ & & $\begin{array}{l}3564 \\
(78.5)\end{array}$ & $\begin{array}{l}976 \\
(21.5)\end{array}$ & $\begin{array}{l}2791 \\
(61.5)\end{array}$ & $\begin{array}{l}1749 \\
(38.5)\end{array}$ & $\begin{array}{l}874 \\
(19.3)\end{array}$ & $\begin{array}{l}3666 \\
(80.7)\end{array}$ & $\begin{array}{l}2384 \\
(52.5)\end{array}$ & $\begin{array}{l}2156 \\
(47.5)\end{array}$ & $\begin{array}{l}2603 \\
(57.3)\end{array}$ & $\begin{array}{l}1937 \\
(42.7)\end{array}$ \\
\hline $\begin{array}{c}\text { Ethiopia } \\
2016\end{array}$ & $\begin{array}{l}9537 \\
(970)\end{array}$ & $\begin{array}{l}290 \\
(30)\end{array}$ & $\begin{array}{l}866 \\
(8.8)\end{array}$ & $\begin{array}{l}89 \\
(9\end{array}$ & & $\begin{array}{l}8099 \\
(82.4)\end{array}$ & $\begin{array}{l}1728 \\
(17.6)\end{array}$ & $\begin{array}{l}8811 \\
(89.7)\end{array}$ & $\begin{array}{l}1016 \\
(10.3)\end{array}$ & $\begin{array}{l}6643 \\
(67.6)\end{array}$ & $\begin{array}{l}3184 \\
(32.4)\end{array}$ & $\begin{array}{l}5363 \\
(54.6)\end{array}$ & $\begin{array}{l}4464 \\
(45.4)\end{array}$ & $\begin{array}{l}8053 \\
(81.9)\end{array}$ & $\begin{array}{l}1774 \\
(18.1)\end{array}$ \\
\hline $\begin{array}{l}\text { Nepal } \\
2016\end{array}$ & $\begin{array}{l}4874 \\
(980)\end{array}$ & $\begin{array}{l}102 \\
(20)\end{array}$ & $\begin{array}{l}760 \\
(15.3)\end{array}$ & $\begin{array}{l}42 \\
(8\end{array}$ & & $\begin{array}{l}2145 \\
(43.1)\end{array}$ & $\begin{array}{l}2831 \\
(56.9)\end{array}$ & $\begin{array}{l}2642 \\
(53.1)\end{array}$ & $\begin{array}{l}2334 \\
(46.9)\end{array}$ & $\begin{array}{l}1123 \\
(22.6)\end{array}$ & $\begin{array}{l}3853 \\
(77.4)\end{array}$ & $\begin{array}{l}2380 \\
(47.8)\end{array}$ & $\begin{array}{l}2596 \\
(52.2)\end{array}$ & $\begin{array}{l}1815 \\
(36.5)\end{array}$ & $\begin{array}{l}3161 \\
(63.5)\end{array}$ \\
\hline $\begin{array}{l}\text { Timor-Leste } \\
2016\end{array}$ & $\begin{array}{l}52640 \\
(984)\end{array}$ & $\begin{array}{l}94 \\
(16)\end{array}$ & $\begin{array}{l}275 \\
(4.8)\end{array}$ & $\begin{array}{l}54 \\
(9\end{array}$ & & $\begin{array}{l}3801 \\
(66.3)\end{array}$ & $\begin{array}{l}1933 \\
(33.7)\end{array}$ & $\begin{array}{l}2229 \\
(38.9)\end{array}$ & $\begin{array}{l}3505 \\
(61.1)\end{array}$ & $\begin{array}{l}1935 \\
(33.7)\end{array}$ & $\begin{array}{l}3799 \\
(66.3)\end{array}$ & $\begin{array}{l}2037 \\
(35.5)\end{array}$ & $\begin{array}{l}3697 \\
(64.5)\end{array}$ & $\begin{array}{l}2290 \\
(39.9)\end{array}$ & $\begin{array}{l}3444 \\
(60.1)\end{array}$ \\
\hline $\begin{array}{c}\text { Indonesia } \\
2017\end{array}$ & $\begin{array}{l}16561 \\
(987)\end{array}$ & $\begin{array}{l}216 \\
(13)\end{array}$ & $\begin{array}{l}801 \\
(4.8)\end{array}$ & & $\begin{array}{l}76 \\
.2)\end{array}$ & $\begin{array}{l}8415 \\
(50.2)\end{array}$ & $\begin{array}{l}8362 \\
(49.8)\end{array}$ & $\begin{array}{l}4294 \\
(25.6)\end{array}$ & $\begin{array}{l}12483 \\
(74.4)\end{array}$ & $\begin{array}{l}675 \\
(4.0)\end{array}$ & $\begin{array}{l}16102 \\
(96.0)\end{array}$ & $\begin{array}{l}7736 \\
(46.1)\end{array}$ & $\begin{array}{l}9041 \\
(53.9)\end{array}$ & $\begin{array}{l}4564 \\
(27.2)\end{array}$ & $\begin{array}{l}12213 \\
(72.8)\end{array}$ \\
\hline $\begin{array}{l}\text { Benin 2017- } \\
\quad 18\end{array}$ & $\begin{array}{l}11632 \\
(975)\end{array}$ & $\begin{array}{l}296 \\
(25)\end{array}$ & $\begin{array}{l}940 \\
(7.9)\end{array}$ & & & $\begin{array}{l}7326 \\
(61.4)\end{array}$ & $\begin{array}{l}4602 \\
(38.6)\end{array}$ & $\begin{array}{l}10066 \\
(84.4)\end{array}$ & $\begin{array}{l}1862 \\
(15.6)\end{array}$ & $\begin{array}{l}4736 \\
(39.7)\end{array}$ & $\begin{array}{l}7192 \\
(60.3)\end{array}$ & $\begin{array}{l}5212 \\
(43.7)\end{array}$ & $\begin{array}{l}6716 \\
(56.3)\end{array}$ & $\begin{array}{l}9110 \\
(76.4)\end{array}$ & $\begin{array}{l}2818 \\
(23.6)\end{array}$ \\
\hline $\begin{array}{l}\text { Pakistan } \\
\text { 2017-18 }\end{array}$ & $\begin{array}{l}12093 \\
(966)\end{array}$ & $\begin{array}{l}430 \\
(34)\end{array}$ & $\begin{array}{l}794 \\
(6.3)\end{array}$ & & $\begin{array}{l}29 \\
7)\end{array}$ & $\begin{array}{l}6994 \\
(55.8)\end{array}$ & $\begin{array}{l}5529 \\
(44.2)\end{array}$ & $\begin{array}{l}8127 \\
(64.9)\end{array}$ & $\begin{array}{l}4396 \\
(35.1)\end{array}$ & $\begin{array}{l}4990 \\
(39.8)\end{array}$ & $\begin{array}{l}7533 \\
(60.2)\end{array}$ & $\begin{array}{l}5731 \\
(45.8)\end{array}$ & $\begin{array}{l}6792 \\
(54.2)\end{array}$ & $\begin{array}{l}5234 \\
(41.8)\end{array}$ & $\begin{array}{l}7289 \\
(58.2)\end{array}$ \\
\hline $\begin{array}{r}\text { Guinea } \\
2018\end{array}$ & $\begin{array}{l}7072 \\
(967)\end{array}$ & $\begin{array}{l}96.7 \\
(33)\end{array}$ & $\begin{array}{l}995 \\
(13.6)\end{array}$ & 63 & & $\begin{array}{l}5374 \\
(73.5)\end{array}$ & $\begin{array}{l}1942 \\
(26.5)\end{array}$ & $\begin{array}{l}6518 \\
(89.1)\end{array}$ & $\begin{array}{l}798 \\
(10.9)\end{array}$ & $\begin{array}{l}2536 \\
(34.7)\end{array}$ & $\begin{array}{l}4780 \\
(65.3)\end{array}$ & $\begin{array}{l}3499 \\
(47.8)\end{array}$ & $\begin{array}{l}3817 \\
(52.2)\end{array}$ & $\begin{array}{l}5931 \\
(81.1)\end{array}$ & $\begin{array}{l}1385 \\
(18.9)\end{array}$ \\
\hline \multirow[t]{2}{*}{2018} & $\begin{array}{l}30169 \\
(964)\end{array}$ & $\begin{array}{l}1140 \\
(36)\end{array}$ & $\begin{array}{l}2922 \\
(9.3)\end{array}$ & & & $\begin{array}{l}20664 \\
(66.0)\end{array}$ & $\begin{array}{l}10645 \\
(34.0)\end{array}$ & $\begin{array}{l}19339 \\
(61.8)\end{array}$ & $\begin{array}{l}11970 \\
(38.2)\end{array}$ & $\begin{array}{l}12659 \\
(40.4)\end{array}$ & $\begin{array}{l}18650 \\
(59.6)\end{array}$ & $\begin{array}{l}14701 \\
(47.0)\end{array}$ & $\begin{array}{l}16608 \\
(53.0)\end{array}$ & $\begin{array}{l}15963 \\
(51.0)\end{array}$ & $\begin{array}{l}15346 \\
(49.0)\end{array}$ \\
\hline & \multicolumn{15}{|l|}{ Variable } \\
\hline \multirow[t]{2}{*}{$\begin{array}{l}\text { Country } \\
\text { Name }\end{array}$} & \multicolumn{2}{|c|}{$\begin{array}{l}\text { Mother's Working } \\
\text { Status n (\%) }\end{array}$} & \multicolumn{3}{|c|}{ Birth Order n (\%) } & \multicolumn{2}{|c|}{$\begin{array}{l}\text { Number of Fetus } \\
\mathrm{n}(\%)\end{array}$} & \multicolumn{2}{|c|}{$\begin{array}{l}\text { Sex of Child } \\
\mathrm{n}(\%)\end{array}$} & \multicolumn{2}{|c|}{$\begin{array}{l}\text { Place of Delivery } \\
\mathrm{n}(\%)\end{array}$} & \multicolumn{2}{|c|}{$\begin{array}{l}\text { ANC Visit } \\
\mathrm{n}(\%)\end{array}$} & \multicolumn{2}{|c|}{$\begin{array}{l}\text { Size of Child } \\
\mathrm{n}(\%)\end{array}$} \\
\hline & $\begin{array}{l}\text { Not } \\
\text { working }\end{array}$ & Working & $\begin{array}{l}1 \mathrm{~s} \\
\text { bo }\end{array}$ & & $\begin{array}{l}\text { 2nd \& } \\
\text { above }\end{array}$ & Single & $\begin{array}{l}\text { One of } \\
\text { multiple }\end{array}$ & Male & Female & Home & Hospital & No & Yes & Average & $\begin{array}{l}>\text { or } \\
<\text { average }\end{array}$ \\
\hline $\begin{array}{l}\text { Sierra Leone } \\
2013\end{array}$ & $\begin{array}{l}2397 \\
(23.6)\end{array}$ & $\begin{array}{l}7767 \\
(76.4)\end{array}$ & $\begin{array}{l}17 \\
(1\end{array}$ & & $\begin{array}{l}8454 \\
(83.2)\end{array}$ & $\begin{array}{l}9710 \\
(95.5)\end{array}$ & $\begin{array}{l}454 \\
(4.5)\end{array}$ & $\begin{array}{l}5088 \\
(50.1)\end{array}$ & $\begin{array}{l}5076 \\
(49.9)\end{array}$ & $\begin{array}{l}4490 \\
(44.2)\end{array}$ & $\begin{array}{l}5674 \\
(55.8)\end{array}$ & $\begin{array}{l}4224 \\
(41.6)\end{array}$ & $\begin{array}{l}5940 \\
(58.4)\end{array}$ & $\begin{array}{l}4152 \\
(40.9)\end{array}$ & $\begin{array}{l}6012 \\
(59.1)\end{array}$ \\
\hline $\begin{array}{l}\text { Zambia 2013- } \\
\quad 14\end{array}$ & $\begin{array}{l}4752 \\
(41.0)\end{array}$ & $\begin{array}{l}6829 \\
(59.0)\end{array}$ & $\begin{array}{l}18 \\
(1\end{array}$ & & $\begin{array}{l}9704 \\
(83.8)\end{array}$ & $\begin{array}{l}11186 \\
(96.6)\end{array}$ & $\begin{array}{l}395 \\
(3.4)\end{array}$ & $\begin{array}{l}5892 \\
(50.9)\end{array}$ & $\begin{array}{l}5689 \\
(49.1)\end{array}$ & $\begin{array}{l}3586 \\
(31.0)\end{array}$ & $\begin{array}{l}7995 \\
(69.0)\end{array}$ & $\begin{array}{l}3896 \\
(33.6)\end{array}$ & $\begin{array}{l}7685 \\
(66.4)\end{array}$ & $\begin{array}{l}6891 \\
(59.5)\end{array}$ & $\begin{array}{l}4690 \\
(40.5)\end{array}$ \\
\hline $\begin{array}{l}\text { Bangladesh } \\
2014\end{array}$ & $\begin{array}{l}3682 \\
(78.1)\end{array}$ & $\begin{array}{l}1031 \\
(21.9)\end{array}$ & $\begin{array}{l}19 \\
(4\end{array}$ & & $\begin{array}{l}2775 \\
(58.9)\end{array}$ & $\begin{array}{l}4651 \\
(98.7)\end{array}$ & $\begin{array}{l}62 \\
(1.3)\end{array}$ & $\begin{array}{l}2431 \\
(51.6)\end{array}$ & $\begin{array}{l}2282 \\
(48.4)\end{array}$ & $\begin{array}{l}2857 \\
(60.6)\end{array}$ & $\begin{array}{l}1856 \\
(39.4)\end{array}$ & $\begin{array}{l}1203 \\
(25.5)\end{array}$ & $\begin{array}{l}3510 \\
(74.5)\end{array}$ & $\begin{array}{l}3173 \\
(67.3)\end{array}$ & $\begin{array}{l}1540 \\
(32.7)\end{array}$ \\
\hline $\begin{array}{c}\text { Cambodia } \\
2014\end{array}$ & $\begin{array}{l}2360 \\
(33.4)\end{array}$ & $\begin{array}{l}4715 \\
(66.6)\end{array}$ & $\begin{array}{l}27 \\
(3\end{array}$ & & $\begin{array}{l}4331 \\
(61.2)\end{array}$ & $\begin{array}{l}6943 \\
(98.1)\end{array}$ & $\begin{array}{l}132 \\
(1.9)\end{array}$ & $\begin{array}{l}3558 \\
(50.3)\end{array}$ & $\begin{array}{l}3517 \\
(49.7)\end{array}$ & $\begin{array}{l}1230 \\
(17.4)\end{array}$ & $\begin{array}{l}5845 \\
(82.6)\end{array}$ & $\begin{array}{l}1562 \\
(22.1)\end{array}$ & $\begin{array}{l}5513 \\
(82.6)\end{array}$ & $\begin{array}{l}3755 \\
(53.1)\end{array}$ & $\begin{array}{l}3320 \\
(46.9)\end{array}$ \\
\hline $\begin{array}{l}\text { Ghana } \\
2014\end{array}$ & $\begin{array}{l}1033 \\
(19.2)\end{array}$ & $\begin{array}{l}4351 \\
(80.8)\end{array}$ & $\begin{array}{l}10 \\
(1\end{array}$ & & $\begin{array}{l}4358 \\
(80.9)\end{array}$ & $\begin{array}{l}5111 \\
(94.9)\end{array}$ & $\begin{array}{l}273 \\
(5.1)\end{array}$ & $\begin{array}{l}2796 \\
(51.9)\end{array}$ & $\begin{array}{l}2588 \\
(48.1)\end{array}$ & $\begin{array}{l}1685 \\
(31.3)\end{array}$ & $\begin{array}{l}3699 \\
(68.7)\end{array}$ & $\begin{array}{l}1664 \\
(30.9)\end{array}$ & $\begin{array}{l}3720 \\
(69.1)\end{array}$ & $\begin{array}{l}1789 \\
(33.2)\end{array}$ & $\begin{array}{l}3595 \\
(66.8)\end{array}$ \\
\hline $\begin{array}{l}\text { Kenya } \\
2014\end{array}$ & $\begin{array}{l}3791 \\
(41.2)\end{array}$ & $\begin{array}{l}5400 \\
(58.8)\end{array}$ & $\begin{array}{l}17 \\
(1\end{array}$ & & $\begin{array}{l}7414 \\
(80.7)\end{array}$ & $\begin{array}{l}8956 \\
(97.4)\end{array}$ & $\begin{array}{l}235 \\
(2.6)\end{array}$ & $\begin{array}{l}4626 \\
(50.3)\end{array}$ & $\begin{array}{l}4565 \\
(49.7)\end{array}$ & $\begin{array}{l}4192 \\
(45.6)\end{array}$ & $\begin{array}{l}4999 \\
(54.4)\end{array}$ & $\begin{array}{l}3097 \\
(33.7)\end{array}$ & $\begin{array}{l}6094 \\
(66.3)\end{array}$ & $\begin{array}{l}5449 \\
(59.3)\end{array}$ & $\begin{array}{l}3742 \\
(40.7)\end{array}$ \\
\hline Lesotho 2014 & $\begin{array}{l}1913 \\
(70.1)\end{array}$ & $\begin{array}{l}816 \\
(29.9)\end{array}$ & $\begin{array}{l}95 \\
(3\end{array}$ & & $\begin{array}{l}1776 \\
(65.1)\end{array}$ & $\begin{array}{l}2656 \\
(97.3)\end{array}$ & $\begin{array}{l}73 \\
(2.7)\end{array}$ & $\begin{array}{l}1342 \\
(49.2)\end{array}$ & $\begin{array}{l}1387 \\
(50.8)\end{array}$ & $\begin{array}{l}693 \\
(25.4)\end{array}$ & $\begin{array}{l}2036 \\
(74.6)\end{array}$ & $\begin{array}{l}607 \\
(22.2)\end{array}$ & $\begin{array}{l}2122 \\
(77.8)\end{array}$ & $\begin{array}{l}1810 \\
(66.3)\end{array}$ & $\begin{array}{l}919 \\
(33.7)\end{array}$ \\
\hline $\begin{array}{l}\text { Chad } \\
\qquad 2014-15\end{array}$ & $\begin{array}{l}10182 \\
(60.8)\end{array}$ & $\begin{array}{l}6562 \\
(39.2)\end{array}$ & $\begin{array}{l}24 \\
(1\end{array}$ & & $\begin{array}{l}14319 \\
(85.5)\end{array}$ & $\begin{array}{l}16292 \\
(97.3)\end{array}$ & $\begin{array}{l}452 \\
(2.7)\end{array}$ & $\begin{array}{l}8497 \\
(50.7)\end{array}$ & $\begin{array}{l}8247 \\
(49.3)\end{array}$ & $\begin{array}{l}13583 \\
(81.1)\end{array}$ & $\begin{array}{l}3161 \\
(18.9)\end{array}$ & $\begin{array}{l}10995 \\
(65.7)\end{array}$ & $\begin{array}{l}5749 \\
(34.3)\end{array}$ & $\begin{array}{l}4727 \\
(28.2)\end{array}$ & $\begin{array}{l}12017 \\
(71.8)\end{array}$ \\
\hline $\begin{array}{l}\text { Afghanistan } \\
2015\end{array}$ & $\begin{array}{l}27575 \\
(89.6)\end{array}$ & $\begin{array}{l}3217 \\
(10.4)\end{array}$ & & & $\begin{array}{l}25107 \\
(81.5)\end{array}$ & $\begin{array}{l}30230 \\
(98.2)\end{array}$ & $\begin{array}{l}562 \\
(1.8)\end{array}$ & $\begin{array}{l}15988 \\
(51.9)\end{array}$ & $\begin{array}{l}14804 \\
(48.1)\end{array}$ & $\begin{array}{l}16378 \\
(53.2)\end{array}$ & $\begin{array}{l}14414 \\
(46.8)\end{array}$ & $\begin{array}{l}20344 \\
(66.1)\end{array}$ & $\begin{array}{l}10448 \\
(33.9)\end{array}$ & $\begin{array}{l}19346 \\
(62.8)\end{array}$ & $\begin{array}{l}11446 \\
(37.2)\end{array}$ \\
\hline $\begin{array}{l}\text { Zimbabwe } \\
2015\end{array}$ & $\begin{array}{l}3073 \\
(59.9)\end{array}$ & $\begin{array}{l}2054 \\
(40.1)\end{array}$ & $\begin{array}{l}12 \\
(2\end{array}$ & & $\begin{array}{l}3877 \\
(75.6)\end{array}$ & $\begin{array}{l}4940 \\
(96.4)\end{array}$ & $\begin{array}{l}187 \\
(3.6)\end{array}$ & $\begin{array}{l}2516 \\
(49.1)\end{array}$ & $\begin{array}{l}2611 \\
(50.9)\end{array}$ & $\begin{array}{l}897 \\
(17.1)\end{array}$ & $\begin{array}{l}4248 \\
(82.9)\end{array}$ & $\begin{array}{l}1378 \\
(26.9)\end{array}$ & $\begin{array}{l}3749 \\
(73.1)\end{array}$ & $\begin{array}{l}2499 \\
(48.7)\end{array}$ & $\begin{array}{l}2628 \\
(51.3)\end{array}$ \\
\hline
\end{tabular}


Table 2 (continued)

\begin{tabular}{|c|c|c|c|c|c|c|c|c|c|c|c|c|c|c|}
\hline & Variable & & & & & & & & & & & & & \\
\hline $\begin{array}{l}\text { Angola 2015- } \\
\quad 16\end{array}$ & $\begin{array}{l}2489 \\
(27.0)\end{array}$ & $\begin{array}{l}6722 \\
(73.0)\end{array}$ & $\begin{array}{l}1488 \\
(16.2)\end{array}$ & $\begin{array}{l}7723 \\
(83.8)\end{array}$ & $\begin{array}{l}8939 \\
(97.0)\end{array}$ & $\begin{array}{l}272 \\
(3.0)\end{array}$ & $\begin{array}{l}4626 \\
(50.2)\end{array}$ & $\begin{array}{l}4585 \\
(49.8)\end{array}$ & $\begin{array}{l}5109 \\
(55.5)\end{array}$ & $\begin{array}{l}4102 \\
(44.5)\end{array}$ & $\begin{array}{l}4877 \\
(52.9)\end{array}$ & $\begin{array}{l}4334 \\
(47.1)\end{array}$ & $\begin{array}{l}5387 \\
(58.5)\end{array}$ & $\begin{array}{l}3824 \\
(41.5)\end{array}$ \\
\hline $\begin{array}{l}\text { India } \\
\qquad 2015-16\end{array}$ & $\begin{array}{l}36328 \\
(82.8)\end{array}$ & $\begin{array}{l}7529 \\
(17.2)\end{array}$ & $\begin{array}{l}16449 \\
(37.5)\end{array}$ & $\begin{array}{l}27408 \\
(62.5)\end{array}$ & $\begin{array}{l}43093 \\
(98.3)\end{array}$ & $\begin{array}{l}764 \\
(1.7)\end{array}$ & $\begin{array}{l}22814 \\
(52.0)\end{array}$ & $\begin{array}{l}21043 \\
(48.0)\end{array}$ & $\begin{array}{l}9630 \\
(22.0)\end{array}$ & $\begin{array}{l}34227 \\
(78.0)\end{array}$ & $\begin{array}{l}17232 \\
(39.3)\end{array}$ & $\begin{array}{l}26625 \\
(60.7)\end{array}$ & $\begin{array}{l}30896 \\
(70.4)\end{array}$ & $\begin{array}{l}12961 \\
(29.6)\end{array}$ \\
\hline $\begin{array}{r}\text { Myanmar } \\
2015-16\end{array}$ & $\begin{array}{l}2133 \\
(47.0)\end{array}$ & $\begin{array}{l}2407 \\
(53.0)\end{array}$ & $\begin{array}{l}1475 \\
(32.5)\end{array}$ & $\begin{array}{l}3065 \\
(67.5)\end{array}$ & $\begin{array}{l}4455 \\
(98.1)\end{array}$ & $\begin{array}{l}85 \\
(1.9)\end{array}$ & $\begin{array}{l}2384 \\
(52.5)\end{array}$ & $\begin{array}{l}2156 \\
(47.5)\end{array}$ & $\begin{array}{l}2951 \\
(65.0)\end{array}$ & $\begin{array}{l}1589 \\
(35.0)\end{array}$ & $\begin{array}{l}1408 \\
(31.0)\end{array}$ & $\begin{array}{l}3132 \\
(69.0)\end{array}$ & $\begin{array}{l}2811 \\
(61.9)\end{array}$ & $\begin{array}{l}1729 \\
(38.1)\end{array}$ \\
\hline Ethiopia 2016 & $\begin{array}{l}7246 \\
(73.7)\end{array}$ & $\begin{array}{l}2581 \\
(26.3)\end{array}$ & $\begin{array}{l}1874 \\
(19.1)\end{array}$ & $\begin{array}{l}7953 \\
(80.9)\end{array}$ & $\begin{array}{l}9563 \\
(97.3)\end{array}$ & $\begin{array}{l}264 \\
(2.7)\end{array}$ & $\begin{array}{l}5071 \\
(51.6)\end{array}$ & $\begin{array}{l}4756 \\
(48.4)\end{array}$ & $\begin{array}{l}6689 \\
(68.1)\end{array}$ & $\begin{array}{l}3138 \\
(31.9)\end{array}$ & $\begin{array}{l}5569 \\
(56.7)\end{array}$ & $\begin{array}{l}4258 \\
(43.3)\end{array}$ & $\begin{array}{l}4129 \\
(42.0)\end{array}$ & $\begin{array}{l}5698 \\
(58.0)\end{array}$ \\
\hline $\begin{array}{l}\text { Nepal } \\
2016\end{array}$ & $\begin{array}{l}2399 \\
(48.2)\end{array}$ & $\begin{array}{l}2577 \\
(51.8)\end{array}$ & $\begin{array}{l}1958 \\
(39.3)\end{array}$ & $\begin{array}{l}3018 \\
(60.7)\end{array}$ & $\begin{array}{l}4909 \\
(98.7)\end{array}$ & $\begin{array}{l}67 \\
(1.3)\end{array}$ & $\begin{array}{l}2623 \\
(52.7)\end{array}$ & $\begin{array}{l}2353 \\
(47.3)\end{array}$ & $\begin{array}{l}2049 \\
(41.2)\end{array}$ & $\begin{array}{l}2927 \\
(58.8)\end{array}$ & $\begin{array}{l}1263 \\
(25.4)\end{array}$ & $\begin{array}{l}3713 \\
(74.6)\end{array}$ & $\begin{array}{l}3307 \\
(66.5)\end{array}$ & $\begin{array}{l}1669 \\
(33.5)\end{array}$ \\
\hline $\begin{array}{l}\text { Timor-Leste } \\
2016\end{array}$ & $\begin{array}{l}3604 \\
(62.9)\end{array}$ & $\begin{array}{l}2130 \\
(37.1)\end{array}$ & $\begin{array}{l}1453 \\
(25.3)\end{array}$ & $\begin{array}{l}4281 \\
(74.7)\end{array}$ & $\begin{array}{l}5624 \\
(98.1)\end{array}$ & $\begin{array}{l}110 \\
(1.9)\end{array}$ & $\begin{array}{l}2973 \\
(51.8)\end{array}$ & $\begin{array}{l}2761 \\
(48.2)\end{array}$ & $\begin{array}{l}2427 \\
(42.3)\end{array}$ & $\begin{array}{l}3307 \\
(57.7)\end{array}$ & $\begin{array}{l}2178 \\
(38.0)\end{array}$ & $\begin{array}{l}3556 \\
(62.0)\end{array}$ & $\begin{array}{l}3988 \\
(69.6)\end{array}$ & $\begin{array}{l}1746 \\
(30.4)\end{array}$ \\
\hline $\begin{array}{c}\text { Indonesia } \\
2017\end{array}$ & $\begin{array}{l}9025 \\
(53.8)\end{array}$ & $\begin{array}{l}7752 \\
(46.2)\end{array}$ & $\begin{array}{l}5459 \\
(32.5)\end{array}$ & $\begin{array}{l}11318 \\
(67.5)\end{array}$ & $\begin{array}{l}16541 \\
(98.6)\end{array}$ & $\begin{array}{l}236 \\
(1.4)\end{array}$ & $\begin{array}{l}8663 \\
(51.6)\end{array}$ & $\begin{array}{l}8114 \\
(48.4)\end{array}$ & $\begin{array}{l}4144 \\
(24.7)\end{array}$ & $\begin{array}{l}12633 \\
(75.3)\end{array}$ & $\begin{array}{l}2802 \\
(16.7)\end{array}$ & $\begin{array}{l}13975 \\
(83.3)\end{array}$ & $\begin{array}{l}9020 \\
(53.8)\end{array}$ & $\begin{array}{l}7757 \\
(46.2)\end{array}$ \\
\hline $\begin{array}{l}\text { Benin 2017- } \\
\quad 18\end{array}$ & $\begin{array}{l}2292 \\
(19.2)\end{array}$ & $\begin{array}{l}9636 \\
(80.8)\end{array}$ & $\begin{array}{l}2387 \\
(20.0)\end{array}$ & $\begin{array}{l}9541 \\
(80.0)\end{array}$ & $\begin{array}{l}11343 \\
(95.1)\end{array}$ & $\begin{array}{l}585 \\
(4.9)\end{array}$ & $\begin{array}{l}6035 \\
(50.6)\end{array}$ & $\begin{array}{l}5893 \\
(49.4)\end{array}$ & $\begin{array}{l}1837 \\
(15.4)\end{array}$ & $\begin{array}{l}10091 \\
(84.6)\end{array}$ & $\begin{array}{l}5203 \\
(43.6)\end{array}$ & $\begin{array}{l}6725 \\
(56.4)\end{array}$ & $\begin{array}{l}6869 \\
(57.6)\end{array}$ & $\begin{array}{l}5059 \\
(42.4)\end{array}$ \\
\hline $\begin{array}{l}\text { Pakistan } \\
\text { 2017-18 }\end{array}$ & $\begin{array}{l}11083 \\
(88.5)\end{array}$ & $\begin{array}{l}1440 \\
(11.5)\end{array}$ & $\begin{array}{l}2971 \\
(23.7)\end{array}$ & $\begin{array}{l}9552 \\
(76.3)\end{array}$ & $\begin{array}{l}12204 \\
(97.5)\end{array}$ & $\begin{array}{l}319 \\
(2.5)\end{array}$ & $\begin{array}{l}6125 \\
(48.9)\end{array}$ & $\begin{array}{l}6398 \\
(51.1)\end{array}$ & $\begin{array}{l}4365 \\
(34.9)\end{array}$ & $\begin{array}{l}8158 \\
(65.1)\end{array}$ & $\begin{array}{l}5635 \\
(45.0)\end{array}$ & $\begin{array}{l}6888 \\
(55.0)\end{array}$ & $\begin{array}{l}9284 \\
(74.1)\end{array}$ & $\begin{array}{l}3239 \\
(25.9)\end{array}$ \\
\hline Guinea 2018 & $\begin{array}{l}2340 \\
(32.0)\end{array}$ & $\begin{array}{l}4976 \\
(68.0)\end{array}$ & $\begin{array}{l}6049 \\
(82.7)\end{array}$ & $\begin{array}{l}1267 \\
(17.3)\end{array}$ & $\begin{array}{l}6983 \\
(95.4)\end{array}$ & $\begin{array}{l}333 \\
(4.6)\end{array}$ & $\begin{array}{l}3771 \\
(51.5)\end{array}$ & $\begin{array}{l}3545 \\
(48.5)\end{array}$ & $\begin{array}{l}3701 \\
(50.6)\end{array}$ & $\begin{array}{l}3615 \\
(49.4)\end{array}$ & $\begin{array}{l}3199 \\
(43.7)\end{array}$ & $\begin{array}{l}4117 \\
(56.3)\end{array}$ & $\begin{array}{l}2702 \\
(36.9)\end{array}$ & $\begin{array}{l}4614 \\
(63.1)\end{array}$ \\
\hline Nigeria 2018 & $\begin{array}{l}10278 \\
(32.8)\end{array}$ & $\begin{array}{l}21031 \\
(67.2)\end{array}$ & $\begin{array}{l}5625 \\
(18.0)\end{array}$ & $\begin{array}{l}25684 \\
(82.0)\end{array}$ & $\begin{array}{l}30138 \\
(96.3)\end{array}$ & $\begin{array}{l}1171 \\
(3.7)\end{array}$ & $\begin{array}{l}15946 \\
(50.9)\end{array}$ & $\begin{array}{l}15363 \\
(49.1)\end{array}$ & $\begin{array}{l}18551 \\
(59.3)\end{array}$ & $\begin{array}{l}12758 \\
(40.7)\end{array}$ & $\begin{array}{l}16628 \\
(53.1)\end{array}$ & $\begin{array}{l}14681 \\
(46.9)\end{array}$ & $\begin{array}{l}16411 \\
(52.4)\end{array}$ & $\begin{array}{l}14898 \\
(47.6)\end{array}$ \\
\hline
\end{tabular}

which we estimated DerSimonian and Laird's pooled effect. ${ }^{25}$ Forest plots were used to display $95 \%$ CI, summary measure, and weight of each study for the most significant determinants. ${ }^{26}$ As a summary measure, we used the Odds Ratio (OR), and all findings were weighted to handle bias due to undersampling and oversampling.

\section{Results}

Table 1 illustrates that according to the analytical evidence, the neonatal mortality rate for Bangladesh in 2014 was 27 deaths per 1000 live births. About $24.7 \%$ of women gave birth to their children as teenagers. The respondents included in the study for the analysis were mostly from rural areas (about 68.1\%) and about $21.9 \%$ of women belonged to the poorest family, while another $19.0 \%$ belonged to a poor family. About $41.5 \%$ of mothers ended up with up to primary level education, $47.1 \%$ completed their secondary level education, and the rest $11.4 \%$ managed to complete their education at a higher level. About $38.4 \%$ of mothers were deprived of having the facility of mass media, while the rest $61.6 \%$ had the facility. About $53.5 \%$ of women's partner completed up to primary level education, while only $15.3 \%$ of women's partner managed to complete their higher level. About $41.1 \%$ of women had one child and about $1.3 \%$ of women gave birth to a twin child, while the rest gave birth to a single child. About $51.6 \%$ of women gave birth to a male child and among the mother, only $21.9 \%$ are working. Place of delivery was home for about $60.6 \%$ of women, and the rest $39.4 \%$ gave birth to their children at the hospital. $74.5 \%$ of women took antenatal care visits during their pregnancy while the rest $25.5 \%$ did not. About $67.3 \%$ of women gave birth to averaged sized child, while the rest $13 \%$ and $19.6 \%$ of women gave birth to larger than average and smaller than average children.

The frequency and percentages of the selected factors for 21 developing countries are displayed in Table 2. Table 3 demonstrated the different influential socio-economic determinants of neonatal mortality in Bangladesh. Neonatal mortality was associated with maternal education with an aOR of $0.591(\mathrm{p} \leq 0.001)$ for the secondary level of education. Neonate with higher educated father was 0.35 times less likely $(\mathrm{p} \leq 0.032)$ to die than the neonate with lower educated father. Individuals from poorer and middle-class households showed a significant influence on neonatal mortality. Neonatal mortality for children from were $0.427(p \leq 0.001)$ and $0.467(p \leq 0.001)$ times less likely for poorer and middle-class household, respectively compare to children from poorest family. Similarly, birth order number had a noticeable impact on neonatal mortality with an adjusted OR of $0.329(\mathrm{p} \leq 0.001)$ with respect
Table 3

Results of the binary logistic regression model affecting socio-economic and demographic factors for neonatal mortality in Bangladesh.

\begin{tabular}{|c|c|c|c|c|c|}
\hline \multirow[t]{2}{*}{ Variables } & \multirow[t]{2}{*}{ B } & \multirow[t]{2}{*}{ aOR } & \multirow[t]{2}{*}{$\mathbf{P}$ - value } & \multicolumn{2}{|c|}{ 95\% C.I.for EXP(B) } \\
\hline & & & & Lower & Upper \\
\hline \multicolumn{6}{|l|}{$\begin{array}{l}\text { Maternal age at delivery } \\
\text { Up to } 19 \text { years (ref) }\end{array}$} \\
\hline $\begin{array}{l}\text { Above } 19 \text { years } \\
\text { Type of place of resident }\end{array}$ & -0.67 & 0.51 & 0.001 & 0.34 & 0.76 \\
\hline \multicolumn{6}{|l|}{ Rural (ref) } \\
\hline \multicolumn{6}{|c|}{ Mother's education level } \\
\hline Up to primary (ref) & & & 0.025 & & \\
\hline Secondary & -0.53 & 0.59 & 0.008 & 0.40 & 0.87 \\
\hline \multicolumn{6}{|l|}{ Mass media } \\
\hline \multicolumn{6}{|l|}{ No (ref) } \\
\hline \multicolumn{6}{|l|}{ Wealth Index } \\
\hline Poorest(ref) & & & 0.000 & & \\
\hline Poorer & -0.85 & 0.43 & 0.000 & 0.28 & 0.66 \\
\hline Middle & -0.76 & 0.47 & 0.005 & 0.28 & 0.80 \\
\hline Richer & -0.04 & 0.96 & 0.888 & 0.55 & 1.68 \\
\hline Richest & -0.068 & 0.93 & 0.856 & 0.45 & 1.95 \\
\hline \multicolumn{6}{|l|}{ Father's education } \\
\hline Up to primary (ref) & & & 0.060 & & \\
\hline Secondary & -0.34 & 0.71 & 0.119 & 0.46 & 1.09 \\
\hline \multirow{2}{*}{\multicolumn{6}{|c|}{ Mother's working status }} \\
\hline & \multicolumn{5}{|c|}{ Working (ref) } \\
\hline $\begin{array}{l}\text { Not working } \\
\text { Birth order number }\end{array}$ & -0.11 & 0.90 & 0.601 & 0.61 & 1.34 \\
\hline \multicolumn{6}{|l|}{ 1st born (ref) } \\
\hline \multicolumn{6}{|l|}{ Number of fetus } \\
\hline \multicolumn{6}{|l|}{ Single (ref) } \\
\hline \multicolumn{6}{|l|}{ Sex of child } \\
\hline \multicolumn{6}{|l|}{ Male (ref) } \\
\hline \multicolumn{6}{|l|}{ Place of delivery } \\
\hline \multicolumn{6}{|l|}{ Home (ref) } \\
\hline \multicolumn{6}{|l|}{ Antenatal care utilization } \\
\hline \multicolumn{6}{|l|}{ No (ref) } \\
\hline \multicolumn{6}{|l|}{ Size of child } \\
\hline Average (ref) & & & & & \\
\hline Larger than average & 0.37 & 1.45 & 0.097 & 0.94 & 2.24 \\
\hline Smaller than average & -0.53 & 0.59 & 0.010 & 0.39 & 0.88 \\
\hline
\end{tabular}

$* \mathrm{~B}=\beta$; CI=Confidence Interval; aOR $=$ Adjusted Odds Ratio; ref $=$ Reference Category. 
Table 4

Random-effects model estimation of OR for Bangladesh and 20 other developing countries.

\begin{tabular}{|c|c|c|c|c|c|c|c|c|c|c|c|c|c|}
\hline \multirow[t]{2}{*}{$\begin{array}{l}\text { Country } \\
\text { Name }\end{array}$} & $\begin{array}{l}\text { Maternal } \\
\text { Age at } \\
\text { Delivery }\end{array}$ & $\begin{array}{l}\text { Place of } \\
\text { Residence }\end{array}$ & $\begin{array}{l}\text { Mother's } \\
\text { Education }\end{array}$ & $\begin{array}{l}\text { Media } \\
\text { Access }\end{array}$ & $\begin{array}{l}\text { Wealth } \\
\text { Index }\end{array}$ & $\begin{array}{l}\text { Father's } \\
\text { Education }\end{array}$ & $\begin{array}{l}\text { Mother's } \\
\text { Working } \\
\text { Status }\end{array}$ & $\begin{array}{l}\text { Birth } \\
\text { Order }\end{array}$ & $\begin{array}{l}\text { Number } \\
\text { of Fetus }\end{array}$ & $\begin{array}{l}\text { Sex of } \\
\text { Child }\end{array}$ & $\begin{array}{l}\text { Place of } \\
\text { Delivery }\end{array}$ & $\begin{array}{l}\text { ANC } \\
\text { Visits }\end{array}$ & $\begin{array}{l}\text { Size of } \\
\text { Child }\end{array}$ \\
\hline & OR & OR & OR & OR & OR & OR & OR & OR & OR & OR & OR & OR & OR \\
\hline Afghanistan & 0.82 & 0.68 & 0.98 & 0.86 & 0.93 & 0.88 & 1.99 & 1.10 & 0.15 & 1.18 & 0.66 & 0.57 & 0.49 \\
\hline Angola & 0.59 & 0.63 & 0.61 & 0.74 & 0.65 & 0.73 & 1.29 & 0.66 & 0.23 & 1.41 & 1.13 & 0.57 & 0.64 \\
\hline Bangladesh & 0.98 & 0.95 & 0.67 & 0.71 & 0.78 & 0.62 & 1.37 & 0.85 & 0.11 & 1.41 & 1.11 & 0.33 & 0.45 \\
\hline Benin & 0.65 & 0.81 & 0.79 & 1.04 & 0.94 & 0.89 & 1.12 & 0.91 & 0.16 & 1.38 & 1.09 & 0.39 & 0.82 \\
\hline Cambodia & 0.93 & 0.54 & 0.90 & 0.90 & 0.54 & 0.75 & 1.75 & 1.17 & 0.33 & 1.23 & 0.65 & 0.29 & 0.57 \\
\hline Chad & 0.57 & 1.01 & 0.98 & 1.21 & 0.94 & 0.98 & 1.21 & 0.60 & 0.18 & 1.57 & 1.24 & 0.53 & 1.29 \\
\hline Ethiopia & 0.70 & 0.51 & 0.85 & 0.68 & 0.73 & 0.66 & 0.99 & 0.94 & 0.17 & 1.90 & 0.85 & 0.33 & 0.65 \\
\hline Ghana & 1.42 & 1.01 & 1.04 & 1.91 & 0.95 & 1.12 & 1.03 & 0.93 & 0.17 & 1.27 & 1.23 & 0.24 & 0.88 \\
\hline Guinea & 0.91 & 0.60 & 0.60 & 1.09 & 0.75 & 0.76 & 1.11 & 0.77 & 0.15 & 1.09 & 0.79 & 0.48 & 0.99 \\
\hline India & 0.64 & 0.75 & 0.59 & 0.69 & 0.60 & 0.63 & 1.13 & 0.97 & 0.12 & 1.30 & 0.73 & 0.38 & 0.62 \\
\hline Indonesia & 0.62 & 1.09 & 0.87 & 0.86 & 0.90 & 0.85 & 1.26 & 1.17 & 10.05 & 2.01 & 0.96 & 0.24 & 0.55 \\
\hline Kenya & 0.71 & 1.01 & 0.96 & 1.15 & 1.01 & 0.95 & 1.20 & 1.06 & 0.15 & 1.26 & 0.85 & 0.32 & 0.41 \\
\hline Lesotho & 1.10 & 0.69 & 1.02 & 0.82 & 0.99 & 0.93 & 0.44 & 0.82 & 0.24 & 1.24 & 0.72 & 0.37 & 0.41 \\
\hline Myanmar & 0.96 & 0.82 & 0.92 & 0.95 & 0.97 & 1.07 & 1.39 & 1.33 & 0.07 & 1.23 & 0.73 & 0.24 & 0.48 \\
\hline Nepal & 0.47 & 0.70 & 0.70 & 1.06 & 0.49 & 0.82 & 0.93 & 0.82 & 0.32 & 1.14 & 0.57 & 0.18 & 0.36 \\
\hline Nigeria & 0.59 & 0.82 & 0.81 & 0.85 & 0.84 & 0.79 & 0.99 & 0.76 & 0.170 & 1.23 & 1.03 & 0.53 & 0.93 \\
\hline Pakistan & 0.87 & 0.80 & 0.70 & 0.94 & 0.75 & 0.75 & 1.58 & 0.91 & 0.25 & 1.33 & 0.88 & 0.51 & 0.59 \\
\hline $\begin{array}{l}\text { Sierra } \\
\text { Leone }\end{array}$ & 0.71 & 1.39 & 1.17 & 1.04 & 0.23 & 1.12 & 0.80 & 0.67 & 0.19 & 1.16 & 0.95 & 0.67 & 0.69 \\
\hline Timor-Leste & 0.62 & 0.67 & 0.82 & 0.90 & 0.97 & 0.79 & 1.00 & 0.89 & 0.09 & 1.44 & 0.67 & 0.56 & 0.45 \\
\hline Zambia & 0.39 & 0.90 & 1.02 & 0.87 & 0.92 & 1.03 & 0.95 & 0.55 & 0.14 & 1.03 & 0.82 & 0.28 & 0.62 \\
\hline Zimbabwe & 0.68 & 0.55 & 0.65 & 0.88 & 0.70 & 0.67 & 0.96 & 0.76 & 0.18 & 1.33 & 0.78 & 0.22 & 0.5 \\
\hline $\mathrm{I}^{2}$ & 46.2 & 64.6 & 61.3 & 57.7 & 70.7 & 55.1 & 73.0 & 61.1 & 95.8 & 36.0 & 65.0 & 86.3 & 82.8 \\
\hline$\tau^{2}$ & 0.026 & 0.032 & 0.031 & 0.021 & 0.033 & 0.019 & 0.047 & 0.028 & 0.728 & 0.008 & 0.028 & 0.094 & 0.067 \\
\hline
\end{tabular}

$\widehat{\tau}^{2}$ : Estimate of between-study variance.

to 2nd and above. Mothers aged above 19 years had a significant influence on neonatal mortality with an aOR 0.510 ( $\mathrm{p} \leq 0.001)$. The variable number of the fetus showed a significant association with neonatal mortality, where the OR for the category' multiple fetuses' is 8.815 ( $\mathrm{p} \leq$ 0.000). Likewise, taking ANC had a significant influence on neonatal mortality, with an OR of $0.206(\mathrm{p} \leq 0.000)$ for the category' $1-4$ visits. The sizes of children had a significant impact on neonatal mortality with an adjusted OR $1.44(\mathrm{p}=0.097)$ with regards to the category 'larger than average'.

In our study, we intended to use the random-effects model as the study showed a high between-study variation. In Table 4 shows the results of the random effect model estimation results of 21 developing countries, and Table 5 shows the summary effect of different explanatory variables.

About $64.6 \%$ of the variation has been observed for the type of place of residence and the overall $\mathrm{OR}$ indicates that individuals residing in urban areas have a $20 \%$ lower chance of experiencing neonatal deaths than their rural counterparts. About $61.3 \%$ of the variation has been found for maternal education and its overall OR demonstrates that, the mothers who have above primary level education are $18.0 \%$ less likely to confront neonatal death compared to the mothers who have up to primary education. Similarly, father's education shows about $55.1 \%$ of the variation with overall OR indicates, with an increment in education level, fathers have 0.830 times or $17 \%$ lower chance of experiencing neonatal death. For living below the poverty line, $\mathrm{I}^{2}$ has been found to be $70.7 \%$ where the overall OR reveals the odds of neonatal mortality is 0.823 times lower to the individuals who do not live below the poverty line compared to those who live below the poverty line. About $73.0 \%$ of the variation has been observed for maternal current working status and the overall OR reveled the odds of neonatal mortality is 1.1388 times higher in the women who are currently working compared to those who are not working currently. For birth order number $\mathrm{I}^{2}$ has been found to

Table 5

Random-effects model estimation (summary effect) for different independent variables in Bangladesh and 21 other developing countries.

\begin{tabular}{|c|c|c|c|c|}
\hline \multirow[t]{3}{*}{ Variables } & \multicolumn{4}{|c|}{ Random effects model } \\
\hline & \multirow[t]{2}{*}{ Odds Ratio (OR) } & \multirow[t]{2}{*}{ P-Value } & \multicolumn{2}{|c|}{ Confidence Interval (CI) } \\
\hline & & & Lower Bound & Upper Bound \\
\hline Maternal age at delivery & 0.68 & 0.0111 & 0.62 & 0.75 \\
\hline Type of Place of Residence & 0.80 & 0.0319 & 0.73 & 0.89 \\
\hline Mother's Education Level & 0.82 & 0.0306 & 0.74 & 0.91 \\
\hline Media Access & 0.91 & 0.0005 & 0.83 & 0.99 \\
\hline Wealth Index & 0.82 & 0.0001 & 0.75 & 0.91 \\
\hline Father's Education Level & 0.83 & 0.0013 & 0.76 & 0.91 \\
\hline Mother's Working Status & 1.14 & 0.0001 & 1.02 & 1.28 \\
\hline Birth Order & 0.86 & 0.0001 & 0.78 & 0.95 \\
\hline Number of Fetus & 0.20 & 0.0001 & 0.14 & 0.29 \\
\hline Sex of Child & 1.32 & 0.0518 & 1.23 & 1.41 \\
\hline Place of Delivery & 0.86 & 0.0001 & 0.79 & 0.95 \\
\hline ANC Visits & 0.37 & 0.0001 & 0.32 & 0.43 \\
\hline Size of Child & 0.61 & 0.0001 & 0.54 & 0.70 \\
\hline
\end{tabular}




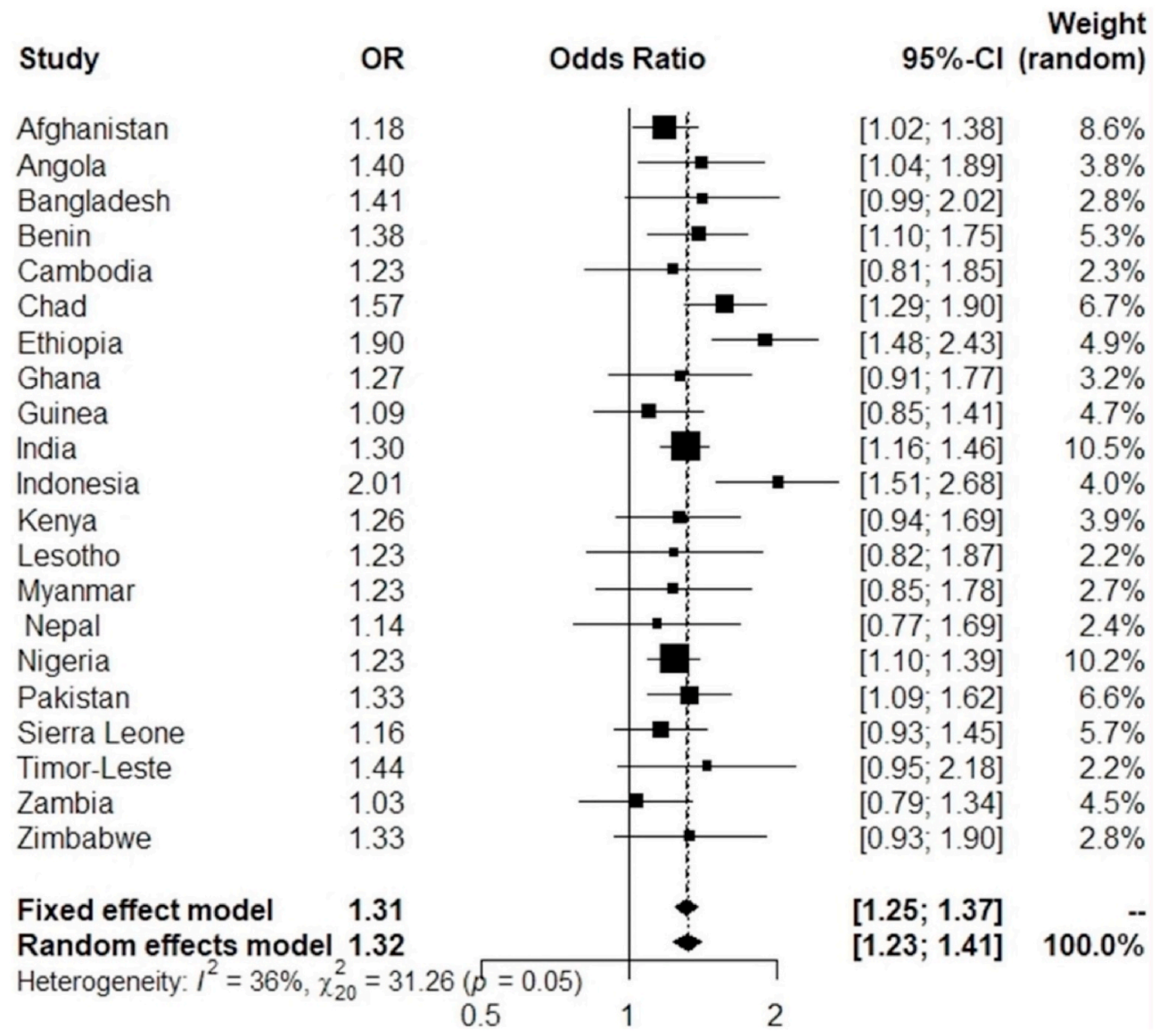

Fig. 2. Forest plot for the sex of the child showing the weight of the study by the size of each box while each crossed line indicates $95 \%$ CI.

be $61.1 \%$ with overall OR 0.862 , indicating $13.8 \%$ lower chance of neonatal mortality in the second and above born child than those who are the firstborn child. The overall OR for maternal age at delivery with a $46.2 \%$ variation. This suggests that with the increment of the mother's age, the risk of neonatal death decreases. Again, the overall OR for the number of the fetus is 0.200 with $72.8 \%$ variation which indicates that neonatal death occurs 0.200 times less likely in the women who carry a single fetus than those women who carry multiple fetuses. About $86.3 \%$ of the variation has been found for taking ANC and the overall OR demonstrates that, the neonatal death occurs 0.374 times less likely in the women who took antenatal care compared to those women who do not take it. Size of child shows $82.8 \%$ variation with overall OR of 0.612 which indicates that neonatal death occurs 0.612 times less likely in the average-sized child compared to those children who are larger or smaller than average. The overall OR of the sex of the child indicates that neonatal death occurs 1.319 times more likely in the male child compared to female children. Media access shows $57.7 \%$ variation with overall OR of 0.908 . This indicates that neonatal death occurs 0.908 times less likely in the family with media access compare to the family with no media access. Place of delivery shows $65 \%$ variation with overall OR of 0.864 . This indicates that neonatal death occurs 0.864 times less for hospital delivery compares to home delivery.

Fig. 2 illustrates that the women of Indonesia have the highest chance of confronting neonatal deaths of male children compare to female children among all the countries, as the OR is 2.01. On the other hand, women of Zambia face an almost equal chance of neonatal death between male and female children. The overall estimate is statistically significant, with a p-value $\leq 0.05$ at a $5 \%$ level of significance.

\section{Discussion}

The third SDG was - "ensure healthy lives and promote well-being for all at all ages." For neonatal death, the target was set as 12 deaths per 1000 live births. To achieve this goal by 2030 , we need to give more focus on developing countries. Although there exists global progress, in sub-Saharan Africa and Southern Asia, child death, especially neonatal death, is increasing comparatively. Out of every five deaths of children under age five four death occurs in these regions.$^{21}$ Our study focused on finding out the socio-economic and demographic factors that affect neonatal death in developing countries. Through the logistic regression model's outcome, maternal education, birth order number, maternal age at delivery, number of fetuses, taking ANC, and child's size, media access was found to have a significant influence on neonatal death for Bangladesh. In this study, the odds of neonatal death is lower among births to mothers who have above primary level education than the mothers with up to primary level education for BDHS data. For instance, mothers who had secondary level education are 0.591 times less likely to confront neonatal death compared to the mothers who had up to primary education. Finding from the meta-analysis for 21 developing countries also supports the fact the maternal education reduces neonatal death. Our study's outcomes are similar to the previous study, and the positive influence of maternal education on neonate survivals is supported worldwide. ${ }^{5,27}$ Both logistic regression models for BDHS data and meta-analysis for 21 developing countries revealed that, like maternal education, the father's higher education reduces the risk of neonatal mortality. Previous studies show an association with paternal education to reduce neonatal mortality, which supports this study's findings. ${ }^{28,29}$ Education increases knowledge about child health and healthcare 
services and improves the healthcare-seeking behaviors for children and themselves. In our society, the father is the main pay worker and chief of the family. His education assumes a job with a significant salary and good earning. Education tends them to live in a rich community with better access to health care services with a short distance. ${ }^{30,31}$ From the outcome of BDHS data, we come to know that the women whose ages were above 19 are 0.510 times less likely to experience neonatal mortality compared to the women whose ages were less or equal to 19 . The meta-analysis also showed a similar association. This result is supported by other study findings, which showed that teenage mothers are more likely to have neonatal death. ${ }^{32-34}$ Lack of childbearing knowledge, awareness, and premature age with different complicacy might be responsible for the higher risk of neonatal mortality to the younger mother. ${ }^{35}$ Binary logistic regression and meta-analysis showed that male children were at higher risk than female children for neonatal mortality. Existing literature and studies confirm these results that male children were more affected by neonatal mortality. ${ }^{5,16,36,37}$ Neonatal mortality is greatly influenced by ANC utilization in developing countries. Results of this study uncovered that neonatal death occurs less likely in the women who took antenatal care compared to those women who did not take it. This result is supported by other studies showing a significant association of ANC with neonatal mortality. ${ }^{16,17}$ Because ANC utilization improves pregnancy outcomes and provides an opportunity for prompt detection of complications and early inception of breastfeeding. With the identification of complications, they get a better chance to resolve the problem, and at the same time, this follow-up with ANC usually leads them to have quality essential newborn cares, which increase survival. ${ }^{38-40}$ Our study showed that children larger than average size were more likely to die in the neonatal period compared to average size children. The result of our study was consistent with previous studies where size of the children influences the rate of neonatal mortality. ${ }^{41,42}$ Logistic regression showed that the second or more birth ordered children were 0.329 times less likely to neonatal death were meta-analysis suggested 0.862 times. The findings of our study were justified by the other studies' results. ${ }^{43}$ The number of fetuses shows a highly significant impact on neonatal mortality. From the outcome of binary logistic regression and meta-analysis estimate, neonatal death occurs likely in the women who carried a single fetus than those women who carried multiple fetuses. Thus, the odds of neonatal death were higher among twin births compared with singleton births. ${ }^{43,44}$ The possible explanation for this finding could be pregnancy with twin fetuses usually lead to prematurity, underweight, which is the most common cause of neonatal death. Additionally, twins' babies create complicacy, which requires emergency and leads to death. ${ }^{45}$

\subsection{Limitation and strength}

We confronted several limitations while conducting the research. We could only collect data from 21 of the 91 countries from the DHS database for missing data, developing countries list, variable availability, etc. For estimating OR from random-effects meta-analysis, we had to create a $2 \times 2$ cross-tabulation for which each variable was categorized into two categories only like maternal age ( $>$ or $<19$ years) and size of the child etc. Moreover, a wide number of factors that could influence neonatal mortality could not be included in our study because of the unavailability of those variables in some DHS data.

The study has some strengths despite these constraints. We combined two methods: binary logistic regression of BDHS data and meta-analysis of 21 DHS data. The integrated findings enlarged the validity of the outcome of the research. We have compared the results of logistic regression analysis and meta-analysis of 21 DHS data. We have used a mixed-method design to generate better knowledge and insights.

\section{Conclusion}

Undoubtedly neonatal mortality is the essential part of under-five child mortality, which reflects social, monetary, and ecological conditions of nations. This study acknowledged the most significant influential factors of neonatal mortality. The number of the fetus, taking ANC and sex of the children, birth order are the dominating factors of neonatal mortality. The utilization of ANC services needs to be ensured and increased across developing countries. Maternal age needs to more than 19, and Parental education needs to be secured to reduce neonatal mortality. Mothers with multiple fetuses should take under proper surveillance. Besides, modernized health care services should be provided mostly to rural areas with free medical facilities for unprivileged counterparts.

\section{Ethical approval}

Not applicable.

\section{Funding}

This research did not receive any specific grant from funding agencies in the public, commercial, or not-for-profit sectors.

\section{Declaration of competing interest}

The authors have no conflicts of interest to declare.

\section{Acknowledgment}

We would also like to show our gratitude to the Demographic and Health Surveys (DHS) Program and the World Bank for providing data access used in this research. The authors also declare the following issues.

\section{References}

1 You D, Hug L, Ejdemyr S, et al. Global, regional, and national levels and trends in under-5 mortality between 1990 and 2015, with scenario-based projections to 2030: a systematic analysis by the UN Inter-agency Group for Child Mortality Estimation. Lancet. 2015;386(10010):2275-2286.

2 Yego F, D’Este C, Byles J, Nyongesa P, Williams JS. A case-control study of risk factors for fetal and early neonatal deaths in a tertiary hospital in Kenya. BMC Pregnancy Childbirth. 2014;14(1):1-9.

3 Organization WH. Newborn Death and Illness. vol. 4. Millennium Development goal (mdg); 2011.

4 Liu L, Oza S, Hogan D, et al. Global, regional, and national causes of under-5 mortality in 2000-15: an updated systematic analysis with implications for the Sustainable Development Goals. Lancet. 2016;388(10063):3027-3035.

5 Titaley CR, Dibley MJ, Agho K, Roberts CL, Hall J. Determinants of neonatal mortality in Indonesia. BMC Publ Health. 2008;8(1):1-15.

6 Målqvist M. Neonatal mortality: an invisible and marginalised trauma. Glob Health Action. 2011;4(1):5724.

7 Devine S, Taylor G, UNICEF. Every Child Alive: The Urgent Need to End Newborn Deaths. Unicef; 2018.

8 de Souza S, Duim E, Nampo FK. Determinants of neonatal mortality in the largest international border of Brazil: a case-control study. BMC Publ Health. 2019;19(1): $1-9$.

9 Jahan S. Poverty and infant mortality in the Eastern Mediterranean region: a metaanalysis. J Epidemiol Community Health. 2008;62(8):745-751.

10 Rahman S, Salameh K, Bener A, El Ansari W. Socioeconomic associations of improved maternal, neonatal, and perinatal survival in Qatar. Int J Wom Health. 2010;2:311.

11 Bhutta ZA, Qadir M. Addressing maternal nutrition and risks of birth asphyxia in developing countries. Arch Pediatr Adolesc Med. 2009;163(7):671-672.

12 Gopalan SS, Das A, Howard N. Maternal and neonatal service usage and determinants in fragile and conflict-affected situations: a systematic review of Asia and the Middle-East. BMC Wom Health. 2017;17(1):1-12.

13 Castro MPd, Rugolo LMSS, Margotto PR. Survival and morbidity of premature babies with less than 32 weeks of gestation in the central region of Brazil. Rev Bras Ginecol Obstet. 2012;34(5):235-242.

14 Choi YY, Song ES, Kim YH, Song TB. Analysis of high-risk infant births and their mortality: ten years' data from chonnam national university hospital. Chonnam J. Med. Sci. 2011;47(1):31.

15 Unicef U. Levels and Trends in Child Mortality. New York: UNICEF; 2015.

16 Bhutta ZA. Community-based primary health care: a core strategy for achieving sustainable development Goals for health. J global health. 2017;7(1).

17 Kumar S, Kumar N, Vivekadhish S. Millennium development goals (MDGS) to sustainable development goals (SDGS): addressing unfinished agenda and 
strengthening sustainable development and partnership. Indian J Community Med: off publ Indian Assoc Preventive \& Soc Med. 2016;41(1):1.

18 Tekelab T, Akibu M, Tagesse N, Tilhaun T, Yohanes Y, Nepal S. Neonatal mortality in Ethiopia: a protocol for systematic review and meta-analysis. Syst Rev. 2019;8(1): $1-4$.

19 National Institute of Population Research and Training, Mitra and Associates, ICF International. Bangladesh Demographic and Health Survey 2014: Key Indicators. Dhaka, Bangladesh, and Rockville. Maryland, USA: NIPORT, Mitra and Associates, and ICF International; 2015.

20 bank W. Countries and Economies; 2019. Available from: https://data.worldbank.or g/country.

21 Hussein J, Hirose A, Owolabi O, Imamura M, Kanguru L, Okonofua F. Maternal death and obstetric care audits in Nigeria: a systematic review of barriers and enabling factors in the provision of emergency care. Reprod Health. 2016;13(1):1-11.

22 Ezeh OK, Agho KE, Dibley MJ, Hall J, Page AN. Determinants of neonatal mortality in Nigeria: evidence from the 2008 demographic and health survey. BMC Publ Health. 2014;14(1):1-10.

23 Cramer JS. The Origins of Logistic Regression. 2002.

24 Huedo-Medina TB, Sánchez-Meca J, Marín-Martínez F, Botella J. Assessing heterogeneity in meta-analysis: Q statistic or $\mathrm{I}^{2}$ index? Psychol Methods. 2006;11(2): 193.

25 DerSimonian R, Laird N. Meta-analysis in clinical trials. Contr Clin Trials. 1986;7(3): $177-188$.

26 Verhagen A, Ferreira ML. Forest plots. J Physiother. 2014;60(3):170-173.

27 Bhutta ZA, Darmstadt GL, Hasan BS, Haws RA. Community-based interventions for improving perinatal and neonatal health outcomes in developing countries: a review of the evidence. Pediatrics. 2005;115(Supplement 2):519-617.

28 Chowdhury QH, Islam R, Hossain K. Socio-economic determinants of neonatal, post neonatal, infant and child mortality. Int J Sociol Anthropol. 2010;2(6):118-125.

29 Singh A, Kumar A, Kumar A. Determinants of neonatal mortality in rural India. PeerJ. 2013;1, e75, 2007-2008.

30 Macassa G, Hallqvist J, Lynch JW. Inequalities in child mortality in sub-Saharan Africa: a social epidemiologic framework. Afr J Health Sci. 2011;18(1-2):14-26.

31 Abuqamar M, Coomans D, Louckx F. The impact of parental education on infant mortality in Gaza strip, Palestine. J Publ Health Epidemiol. 2011;3(1):28-33.

32 Huda TM, Tahsina T, Arifeen SE, Dibley MJ. The importance of intersectoral factors in promoting equity-oriented universal health coverage: a multilevel analysis of social determinants affecting neonatal infant and under-five mortality in Bangladesh. Glob Health Action. 2016;9(1), 29741.

33 Jehan I, Harris H, Salat S, et al. Neonatal mortality, risk factors and causes: a prospective population-based cohort study in urban Pakistan. Bull World Health Organ. 2009;87:130-138.

34 Chen X-K, Wen SW, Fleming N, Yang Q, Walker MC. Increased risks of neonatal and postneonatal mortality associated with teenage pregnancy had different explanations. J Clin Epidemiol. 2008;61(7):688-694.

35 Schell CO, Reilly M, Rosling H, Peterson S, Mia Ekström A. Socioeconomic determinants of infant mortality: a worldwide study of 152 low-, middle-, and highincome countries. Scand J Publ Health. 2007;35(3):288-297.

36 Shakya K, McMURRAY C. Neonatal mortality and maternal health care in Nepal: searching for patterns of association. J Biosoc Sci. 2001;33(1):87.

37 Alonso V, Fuster V, Luna F. Causes of neonatal mortality in Spain (1975-98): influence of sex, rural-urban residence and age at death. J Biosoc Sci. 2006;38(4):537.

38 Bale J, St oll BJ, Lucas AO. Improving Birth Outcomes Meeting the Challenges in the Developing World. Washington, DC: National Academies Press; 2003.

39 Phukan D, Ranjan M, Dwivedi L. Impact of timing of breastfeeding initiation on neonatal mortality in India. Int Breastfeed J. 2018;13(1):1-10.

40 Rarani MA, Rashidian A, Khosravi A, Arab M, Abbasian E, Morasae EK. Changes in socio-economic inequality in neonatal mortality in Iran between 1995-2000 and 2005-2010: an Oaxaca decomposition analysis. Int J Health Pol Manag. 2017;6(4): 219.

41 Akinyemi JO, Bamgboye EA, Ayeni O. Trends in neonatal mortality in Nigeria and effects of bio-demographic and maternal characteristics. BMC Pediatr. 2015;15(1): $1-12$.

42 Kozuki N, Lee AC, Silveira MF, et al. The associations of parity and maternal age with small-for-gestational-age, preterm, and neonatal and infant mortality: a metaanalysis. BMC Publ Health. 2013;13(3):1-10.

43 Kamal SM, Ashrafuzzaman M, Nasreen S. Risk factors of neonatal mortality in Bangladesh. J Nepal Paediatr Soc. 2012;32(1):37-46.

44 Alam N, Van Ginneken JK, Bosch AM. Infant mortality among twins and triplets in rural Bangladesh in 1975-2002. Trop Med Int Health. 2007;12(12):1506-1514.

45 Justesen A, Kunst A. Postneonatal and child mortality among twins in Southern and Eastern Africa. Int J Epidemiol. 2000;29(4):678-683. 